\title{
Listening in to the Cell
}

\author{
Jonathan F. Ashmore, PhD
}

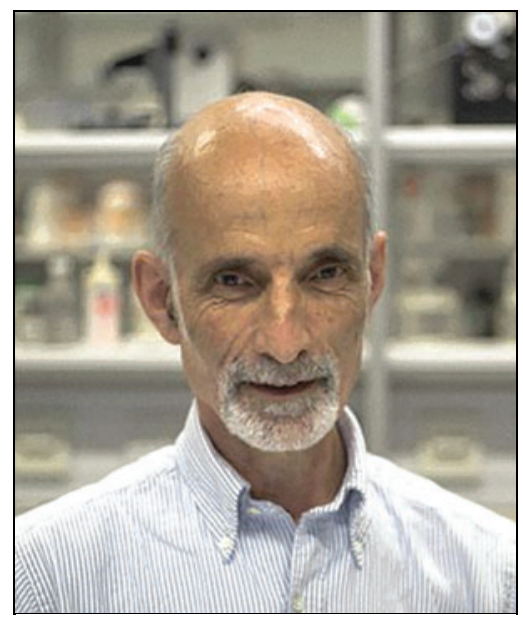

Jonathan F. Ashmore is Bernard Katz Professor of Biophysics at University College London. Here, he discusses his career-changing experiences and experiments in bioelectricity.

\section{Adding the Prefix: From Physics to Biophysics}

Looking up one day from his copy of Pakistan News in his office at Imperial College London, Abdus Salam asked me: "Well what are you doing after your PhD?" I replied that I hadn't even thought about it. Two weeks later, I was a postdoc at the Institute for Theoretical Physics in Trieste. Salam, the first Muslim to win the Nobel Prize for Physics for his work on supersymmetry, had set up the Institute primarily to enable researchers in developing countries to keep in contact with new frontiers. Although my contract was short, it was an exciting time as it was realized that the Higgs boson was a renormalizable (read "respectable") theory, although we all knew that the experiments were decades away (I judged that correctly)! I liked being caught up in some of the technical problems, but I realized pretty soon that I was not cut out to be a deep physics thinker, I just did not know enough. I had undertaken a degree, and then a $\mathrm{PhD}$, in theoretical physics as it seemed difficult and challenging. But the subject was really a flight from what I had spent most of schooldays doing, electronics and small engineering projects.

\section{An Introduction to Thinking About Bioelectricity}

In the 1970s, the Nuffield Foundation had a scheme to try to recruit physicists to biology. There are now many pro- grams that encourage this, but at the time it was an enlightened approach. Believing that biology could use some more mathematics, a year on I attended lectures on genetics and neurobiology, subjects full of words I did not understand and interlocking webs of ideas. At some point during the year, I encountered the Hodgkin-Huxley model of the nerve action potential and was astonished how very arbitrary the model seemed to be. It did not really have the feel of a physics model. I do not know whether this is initial experience of many physicists. The defining equations seemed to be pulled out of thin air, and it was not clear why any of the exponential curves used to fit the data should be there at all. No conclusion seemed to be derived from first principles and the whole style seemed to be very different from the linear thinking of the physical sciences.

Later that year, I retreated back to Trieste for a conference on mathematical biology. During a talk about a very elaborate chemical scheme to explain neural conduction, a member of the audience got up and started shouting that there was absolutely no evidence for the theory: "You cannot get the electrode through the myelin," he bellowed. I thought it was wonderful that in biology people expressed such vehement views, physicists were far more restrained. This was my introduction to Paul Fatt and his wife Gertrude Falk. As a PhD student with Bernard Katz in the early 1950s, Paul had

Department of Neuroscience, Physiology and Pharmacology and UCL Ear Institute, UCL, London, United Kingdom. 
discovered miniature synaptic potentials. In London, I took his biophysics course, six nonstop laboratory weeks of recording from the neuromuscular junction, hard work, but one of the best of introductions to electrophysiology!

\section{The Retina and the Single Photon Signal}

As a result, I became a member in mid-1970s of the UCL Biophysics Department, one of the major centers of physiological biophysics founded by A.V. Hill and then headed by Katz. Paul and Gertrude wanted to understand the origin of the b-wave of the electroretinogram (the electrical signal elicited by light, which can be recoded simply by putting an electrode on the cornea) and to explain how it could be such a sensitive marker of excitation. Much of my equipment had to be built as the technology for recording membrane potentials reliably from small cells was just getting going. Inspired by the work of Alan Hodgkin, Denis Baylor, and Trevor Lamb at Cambridge who had been recording from vertebrate photoreceptors, ${ }^{1}$ the project needed very fine microelectrodes, with resistances over $200 \mathrm{M} \Omega$. We used an electrode puller that required so much force to load the spring that it eluded Gertrude (a handy tip if you wish to discourage your supervisor from looking over your shoulder in the laboratory). I built a lot of the high-impedance electronics, the manipulators and light stimulator to record from retinal cells, and within a year, we were beginning to record from bipolar cells in the isolated retina of dogfish. Everything had to be done in the dark to make sure that we were only getting signals from the rod photoreceptors when light was projected onto the retina surface. The room also had to be quite cold, unpleasant for long periods even in summer, or else the tissue did not survive for long.

The first surprise was that most of the bipolar cells depolarized when illuminated with a short very dim light flash. This remained a puzzle for the next decade, well after I left the laboratory, for it was known that in the dark photoreceptors released neurotransmitter continuously and light suppressed this release. Since most known transmitters opened receptor channels there was a paradox, as the response seemed to be behaving in a "non-classical" way.
(There are also bipolar cells that hyperpolarize, following the neurotransmitter release, and much more understandable.)

The second surprise was that the responses fluctuated. Since the 1940s, it had been known that a rod photoreceptor could respond to the absorption of a single photon. Could the bipolar cells also be collecting information from single photon hits? I tried for a while to build an analog device to analyze the fluctuations, but eventually resigned myself to learning how to program an enormous computer that Katz and Ricardo Miledi had been using to analyze noise fluctuations of the acetylcholine receptor. The PDP8 computer, made by the now deceased Digital Equipment Corporation (DEC), was about the size of a large kitchen dresser and had to be programmed in very low-level code. It was a question of booking the computers for short periods between Katz and Miledi, often in the middle of the night and analyzing our data. The result was clear-bipolar cells could detect a single photon absorption, the response fluctuation arising from the statistics of the quantal nature of light (Fig. 1).

\section{Hearing from the Bottom Up}

Everyone says that so much of science discovery is serendipity. I did not think it true, but reluctantly I now think it is. Ian Russell and Peter Sellick had just made the first intracellular recordings from hair cells in a mammalian cochlea ${ }^{2}$ and were visiting the University of California, San Francisco (UCSF) in San Francisco where I was then a postdoc (my third) as the best intracellular microelectrode puller was being developed there. It was the machine that eventually formed the cornerstone of Sutter Instruments. Ian and I knew each other a little from my time as a Nuffield fellow and so I was invited to take up his position as a temporary lecturer at Sussex University while he enjoyed research leave. Weary of sitting in dark rooms, I thought this a great opportunity to learn about hearing.

A few laboratories with experience of intracellular recording in the retina were beginning to look at other sensory systems. First on the list was to understand how the sensory hair cells of the cochlea could be working. All the technologies for recording electrical signals were there, it was just a
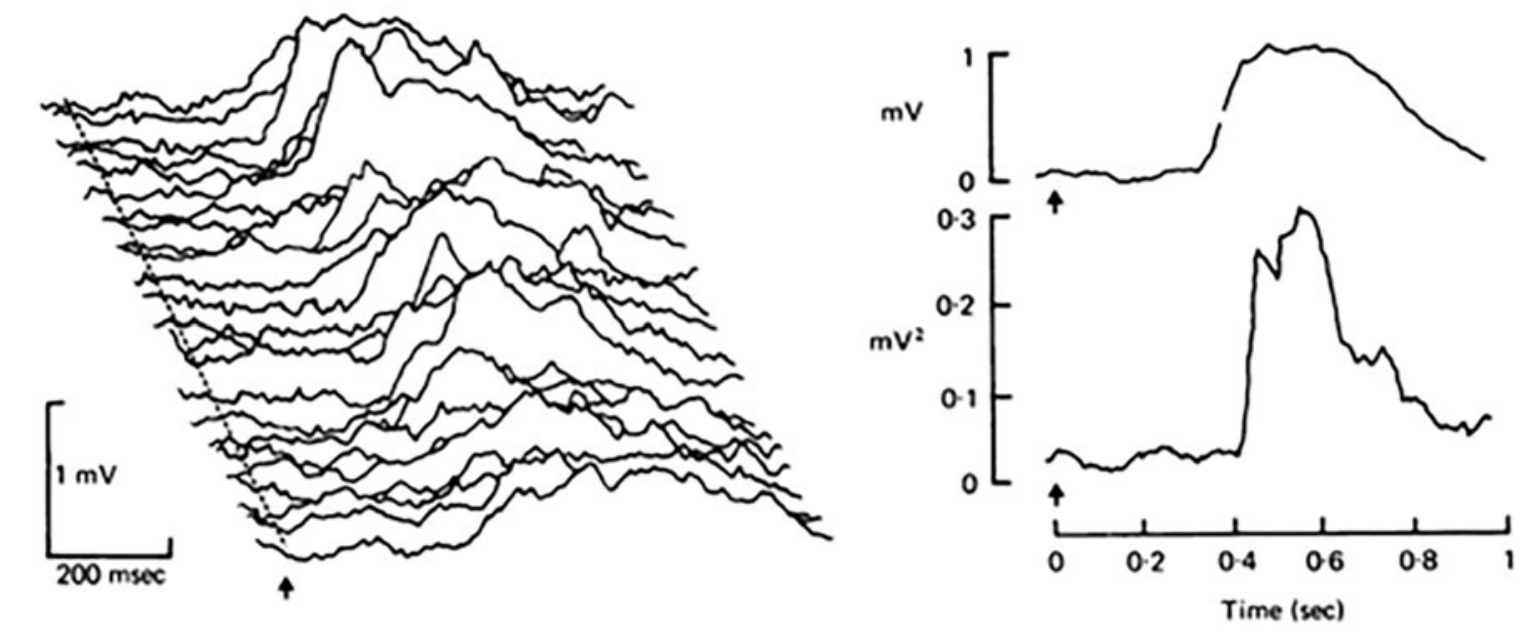

FIG. 1. Fluctuations in response to dim light flashes (arrowed) of a rod bipolar cell. The means and ensemble variance, shown to the right, allow an estimate of the responses to a single photon event. From Ashmore and Falk with permission. ${ }^{12}$ 
question of using it correctly. Crawford and Fettiplace ${ }^{3}$ at Cambridge had started to record electrical signals from turtle hair cells. Corey and Hudspeth ${ }^{4}$ at $\mathrm{Cal}$ Tech had shown how to measure electrical signals from frog hair cells.

After building the microelectrode recording equipment yet again (although to begin with, for lack of funds, some of the items were scavenged from the Brighton rubbish dump), it was possible to record from hair cells in the frog. These cells are quite robust and can be dissected out as an epithelium to be pinned down under microscope objective. The Medical Research Council surprisingly awarded me an equipment grant with an impressively short turnaround time (I suspect that it was realized that Sussex was becoming a center of excellence). The problem was that nobody understood then how the mammalian cochlea could be mechanically tuned to produce high-frequency selectivity in the auditory nerve fibers. Ian and I thought at the time that frog hair cells would be a good model system, technically approachable, to explore basic cochlear principles. Although the recordings were possible and I showed that frog hair cells could underpin a particular type of auditory tuning mechanism, it dawned on us that this system was not the way to understand how mammals hear, as the tuning range of the frog hair cells was relatively low frequency. Different high-frequency mechanisms must be operating in mammals (Fig. 2).

\section{Cochlear Amplification and Outer Hair Cells}

At the beginning of the 1980s, a number of separate results merged to change the way in which cochlea physiology was understood. Although it was known that cochlear auditory nerve fibers were highly frequency selective (so that each fiber responded to only a single pure tone), the mechanism was unknown. The in vivo intracellular recording by Russell and Sellick from inner hair cells, the presynaptic source of auditory nerve fibers, showed that the selectivity was a property of the cochlea rather than some subsequent neural enhancement. The sticking point was that the mammalian cochlea is a fluid-filled coiled tube, and the effects of fluid viscosity should have mechanically dissipated any sound vibration.

We now know that a subpopulation of cochlear cells, outer hair cells (OHCs), behave like ultrafast actuators. OHCs can undo the viscous dissipative damping that would be expected around the basilar membrane. The decisive evidence came in 1985 from a group in Geneva, which showed that OHCs changed length when their membrane potential changed. Many cells can be stimulated to change shape on electrical stimulation (muscle cells for example), but this phenomenon was clearly different. The OHC mechanism was robust and depended on membrane potential. OHC motility seemed to explain how an electrical signal could be transformed to alter the vibration pattern of the basilar membrane.

Unlike the retina, cell biology and cell biophysics were quite late coming to hearing. Much of the impetus had come from Swedish groups centered around the Karolinska Institute, primarily ENT clinicians. They were the first to start looking at the elegant inner ear structures using electron microscopy. Ian Russell, Jim Hudspeth, and Bill Brownell had all spent time in Ake Flock's laboratory in Stockholm. Flock realized the significance of the $\mathrm{OHC}$ population and had rediscovered a way of microdissecting individual hair cells that had first been described in the 1920s. This was the key. Ian and I visited Stockholm with whole suitcases of electrical recording equipment and managed to push a microelectrode into an isolated OHC. It had no membrane potential to speak of, but we occasionally found that the cell seemed to jump apart when impaled. We thought not much of that. Brownell, working with the group in Geneva, was better equipped and had the willingness to look at isolated cells (anathema to a retina biophysicist when everything was done in the dark). He spotted that the cells moved. In hindsight, the recordings from OHCs made back at Sussex showed that the cells moved on current injection (usually off the end of the microelectrode), but the significance escaped us.

\section{Patch-Clamp Recording from Hair Cells}

The first successful recording of the flickering of a single ion channel, the acetylcholine receptor, by Neher and Sakmann in 1976 was an eye opener for biophysics. ${ }^{5}$ The influential article, describing the main methods of patch-clamp recording, came later. Although single-channel recording remains one of the highest resolution single-molecule methods in biology, it is probably whole-cell recording, where the measured current flows through all the channels in a cell, which has yielded the most information. Whole-cell recording allows currents to be measured with a bandwidth
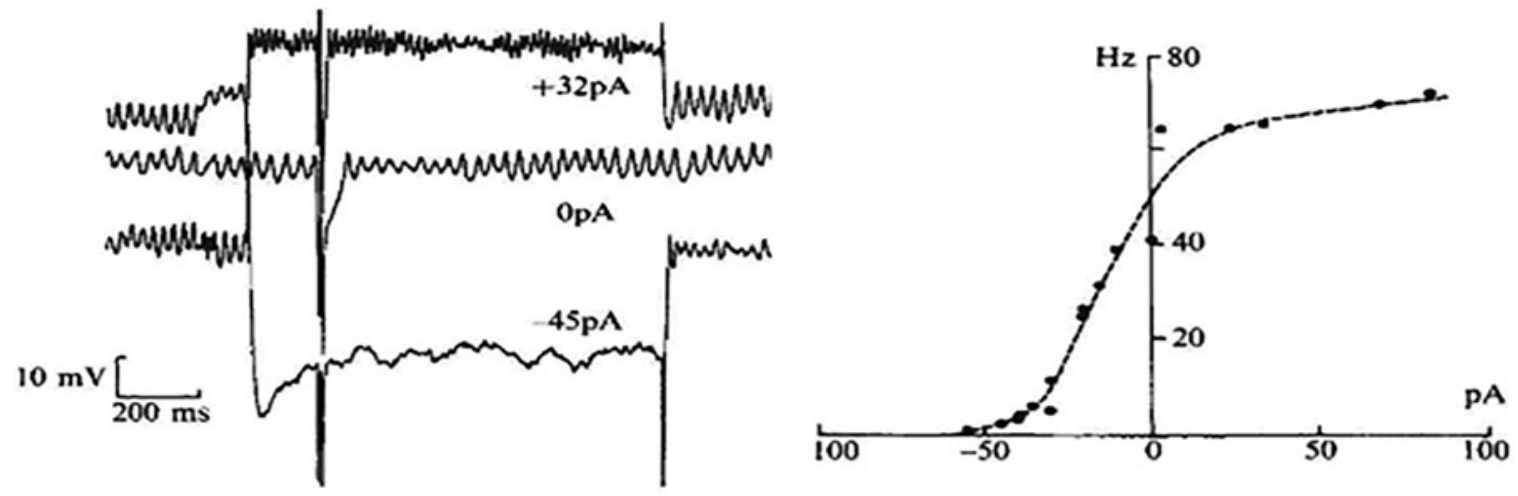

FIG. 2. Spontaneous membrane potential oscillations in a frog saccular hair cell recorded with an intracellular electrode. The oscillation frequency is a function of the potential determined by injected current but allows the cell to respond to low frequency movements. The frequency does not exceed $80 \mathrm{~Hz}$. From Ashmore with permission. ${ }^{13}$ 


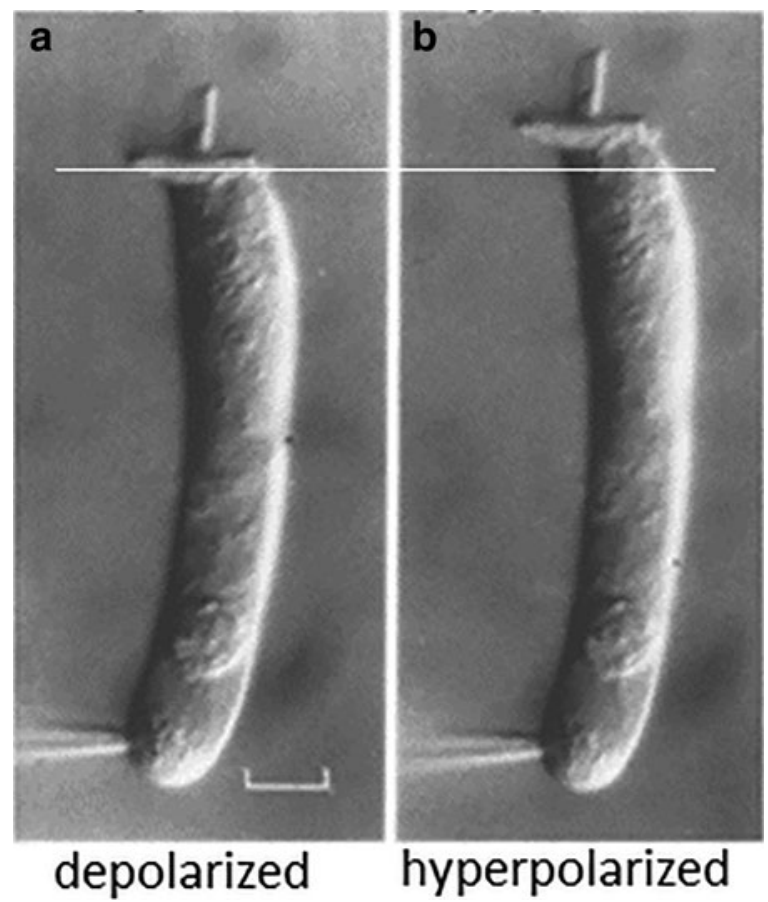

FIG. 3. One of the longer OHCs from the guinea pig cochlea shown with a recording pipette at the base to (a) depolarize or (b) hyperpolarize the cell. The sensory hair bundle is at the apex of the cell. The length change, maximally $4 \%$, is maintained for the duration of the stimulus allowing this photograph to be taken. Scale bar, $8 \mu \mathrm{m}$. From Holley and Ashmore (unpublished). OHC, outer hair cell.

$10 \mathrm{kHz}$, much better than with a sharp microelectrode. Beyond that limit, one has to make many corrections.

In Bristol, Rob Meech and I started to record from guinea pig OHCs. We reported single potassium channels from isolated $\mathrm{OHCs},{ }^{6}$ but it was the advent of good commercial patch-clamp amplifiers in the mid-1980s, which opened up the possibilities for measuring ionic currents using whole-cell recording. At the same time, much of the software for controlling the amplifier and analyzing the electrophysiological data had improved considerably, so it was no longer completely necessary to write an application for every new experiment. Despite the limited bandwidth for membrane potential control, I demonstrated that the patch clamp could extended Brownell's observations to show that OHCs could change length fast enough to be involved in acoustic processes (Fig. 3).

\section{Ionic Currents of OHCs}

$\mathrm{OHCs}$ are long cylindrical cells and can be more than $70 \mu \mathrm{m}$ long in some cases, particularly when dissected from the apical end of the cochlea (which detects low-frequency sound). The trick that we discovered, following Flock's protocol to isolate cells, was to patch the cells at the base where the membrane is more fluid. The lateral sides of the cells were hard to patch well as the surface was densely packed with protein. From the start, we had worked with guinea pig OHCs: these were mature adult cells, and at the time, the guinea pig was a main animal of choice for audi- tory physiology. Much of what we know of mammalian hair cell biophysics has since come from early-stage mouse (postnatal days P0-P14) as the tissue is easier to dissect. Mouse genetic manipulation is an added plus. Nevertheless, developmental changes in the mouse are often not complete, and some features of early-stage cells are not representative of the mature cochlea.

One question was how the electrical properties of the cells varied from one end of the cochlea to the other. To our surprise, not all hair cells expressed the same currents and to the same extent. The $\mathrm{OHC}$ properties varied along the cochlea coil. Long apical cells expressed a calcium-activated potassium current $\left[\mathrm{I}_{\mathrm{K} \text { (ca) }}\right]$, whereas the shorter OHCs expressed a surprising novel $\mathrm{K}^{+}$current, which we called $\mathrm{I}_{\mathrm{Kn}}$ as it activated at a negative potential $(-80 \mathrm{mV})$, below the cell resting potential (Fig. 4). ${ }^{7}$ We now know that $I_{K n}$ arises from the channel KCNQ4 (alternatively named Kv7.4) in the same KCNQ family as those implicated a variety of clinical symptoms ranging from epilepsy to cardiac problems.

\section{Gating Charges and the Molecular Basis of OHC Motility}

The molecular "motor" that makes OHCs move was eventually identified in 2000 by a group at Northwestern University at Evanston using a subtraction DNA library for hair cells. 8 They called the molecule "prestin" as it made the hair cell move "presto." The name prestin stuck even though it was soon found that the molecule was a member number A5 of a family SLC26 of anion-bicarbonate transporters. How could a transport protein, normally thought of as having slow kinetics, act to distort cell membranes?

I had found a hint of this a decade earlier. To record ionic currents with a patch clamp, it is necessary to minimize current that flows to charge the membrane capacitance. The curiosity about $\mathrm{OHCs}$ was that this capacitance compensation was very tricky and had to be redone at each holding potential. I ran down the corridor (a rare event) to show Meech when I realized that on the screen was evidence of an underlying gating charge movement, usually evidence of a molecular reorganization. Since capacitance $C=d Q / d V$, the anomalous capacitance was due to an additional moving charge as well as the charge required for the membrane capacitance itself. This so-called "nonlinear capacitance" (NLC) became an electrical fingerprint for the identification of prestin. The Northwestern group used the NLC in a transfected cell as a biomarker for prestin.

There are so many copies of prestin per OHC that Jonathan Gale and I managed to measure the gating charge in small patches of OHC membrane. All along the side of an $\mathrm{OHC}$, there was a small gating charge movement, matching the movement of the membrane. Even though small, the gating current was sufficiently stable and rapid to be able to pull it out of the noise with averaging the data a few thousand times (Fig. 5).

The only point we missed was that the charge was carried by chloride inside the cell. But that needed the molecular identity of prestin to be known. Prestin/SLC26A5 was eventually expressed and analyzed in $\mathrm{CHO}$ cells by Dominik Oliver and his colleagues at Freiburg. ${ }^{9}$ They showed that the charge movement could largely be explained if intracellular chloride ions were shuttled through the cytoplasmic vestibule 
A
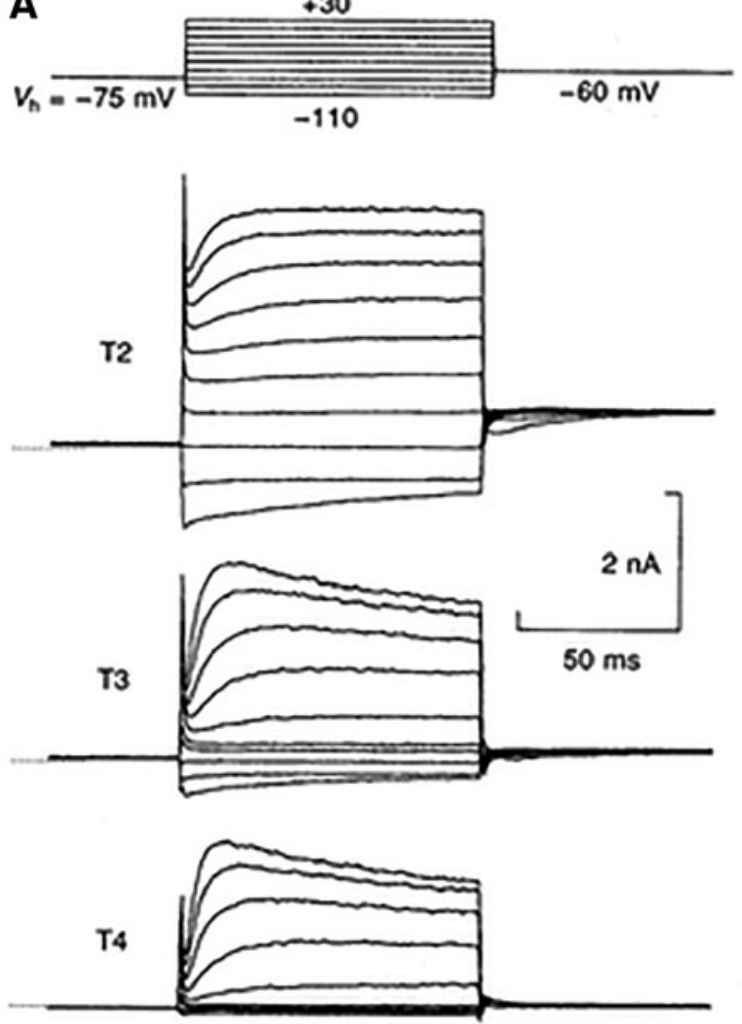

B

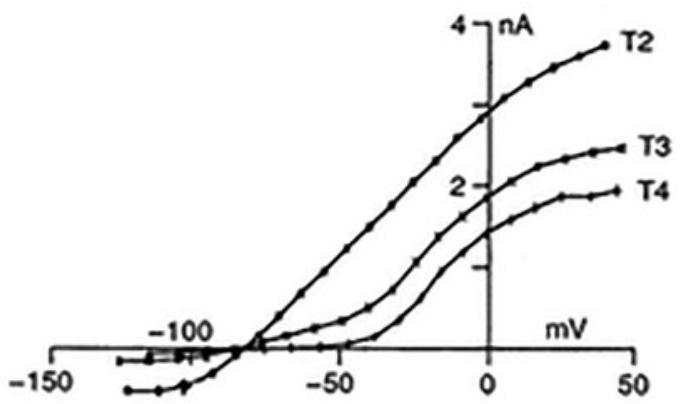

C

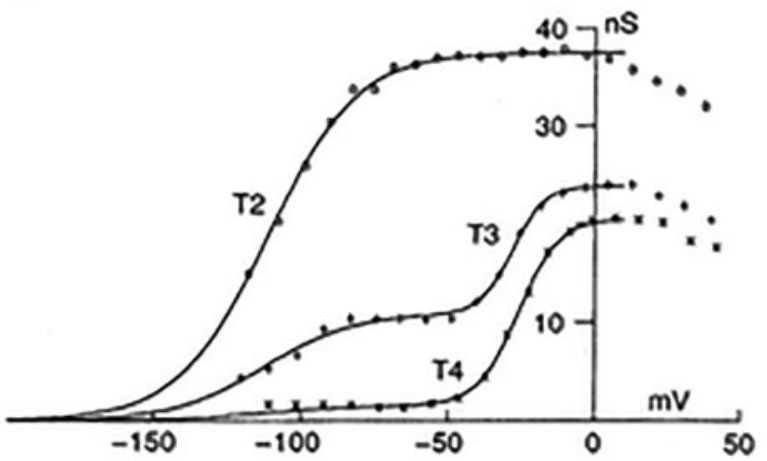

FIG. 4. OHC currents measured with a whole-cell patch clamp when stepped from a holding potential of $-75 \mathrm{mV}$, for various turns of the guinea pig cochlea (A). Turn 4 (T4) is the most apical (low frequency) end, turn2 (T2) is the penultimate basal turn. The initial deactivating current evident in T2 is $\mathrm{I}_{\mathrm{Kn}}$ (KCNQ4). Shorter cells from the cochlear base also have (B) larger currents and (C) larger conductances. From Mammano and Ashmore with permission. ${ }^{14}$

of the molecule but only about $70 \%$ of the way through the membrane leaving the transport cycle incomplete. This would look like a gating charge movement. The hypothesis was (and still is to a large extent) that as chloride moves into the electric field the molecule expands, by about $4 \%$ in the plane of the membrane. As a result of the close packing of prestin, the $\mathrm{OHC}$ elongates.

We still do not know the precise structure of prestin. Nevertheless, it is possible to show that prestin is a low efficiency antiporter, exchanging bicarbonate for chloride across the membrane. ${ }^{10}$ The other surprising finding is that
SLC26A5 homologs are found in all hair cells, vertebrate and invertebrate, but not at the same density as in mammalian OHCs. The conclusion, although speculative, is that SLC26A5 homologs provide a conserved $\mathrm{pH}$ regulation system in sensory hair cells. It is just that mammalian OHCs have found an additional role for the protein.

\section{Cochlear Synapses and Confocal Imaging}

Although it became routine to record the ionic currents from single cells, the site of the current flow, possibly at
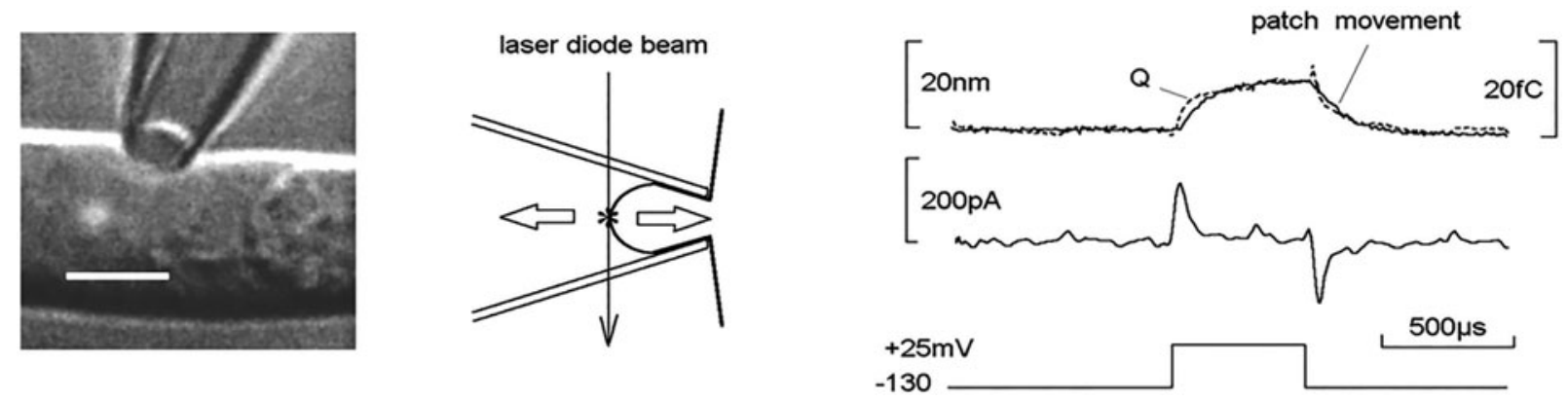

FIG. 5. The gating charge from a small patch of lateral $\mathrm{OHC}$ membrane. By measuring the local patch displacement with a laser beam, the patch movement tracks the total charge, Q, moved. Scale bar, $5 \mu \mathrm{m}$. From Gale and Ashmore with permission. ${ }^{15}$ 
FIG. 6. The effect of Wnt peptide on a PC3 prostate cancer cell: a biphasic response. Adding a calcium indicator (OGB5N) to the pipette solution allows intracellular calcium to be measured at the same time as the cell potential, while adding a neutral dye (Alexa647) to the Wnt pipette monitors the peptide dose and application delivery. Modified from Ashmore et al. ${ }^{16}$
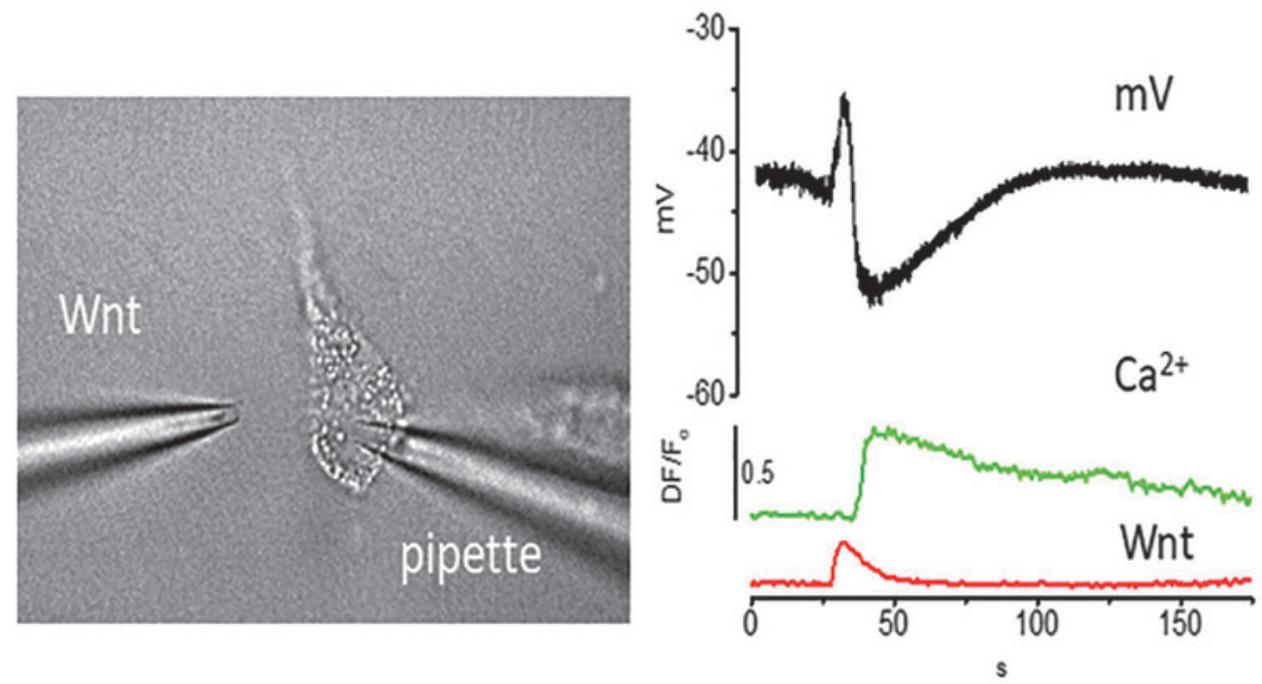

multiple sites, and the link to the internal signaling pathways become important. The use of calcium-sensitive dyes and single-cell imaging has thus been added to many electrical measurements. In neuroscience, calcium signaling at the neuronal synapses is critical. Very often the calcium currents themselves are quite small. In the cochlea, the inner hair cells form multiple synaptic contacts, not all equivalent, with the auditory nerve. The latter became the next focus of the laboratory, preferably exploiting the techniques to work with adult hair cell systems.

We were lucky enough to have almost exclusive access to a multiphoton confocal microscope, and thus, adult inner hair cell synaptic release could be investigated. ${ }^{11}$ Adding patchclamp recording to the system becomes a synchronization problem to match the imaging with electrophysiology but with submillisecond accuracy. Such improvements expand the types of possible experiment. This system has allowed us to look at multiple sites of calcium entry in inner hair cells during membrane control by the patch pipette.

\section{Recording Electrical Signals in Cancer}

Many of the techniques for recording electrical signals from cells can be translated to other systems once the tricks to work with new tissues are developed. Five years ago, a chance conversation with Aamir Ahmed from Kings College London turned into another project. He had been working on a prostate cancer cells, imaging calcium but not the electrical signals and had shown that Wnt peptides initiated a burst of calcium in the cells and subsequent proliferation. Wnts are familiar from developmental biology, where gradients of the peptide determine cell division and organization. The idea that some Wnts could trigger tumors, particularly in prostate and breast tissues, was intriguing and possibly useful for designing treatments.

Aamir had collaborated with a biotech company, Sophion, in Denmark that makes high-throughput, multiwell patchclamp machines. These machines are designed primarily not as discovery but as drug screening stands. He wanted to see whether a "classical" patch clamp, a slightly insulting term for a single micropipette, gave the same answers. We began a slow collaboration-slow because there was about one experiment every fortnight-but discovered how to patch the cells and image the calcium signals at the same time. The technical problem with the Wnts is that the receptor sensitivity is in the nanomolar range. Thus, any leakage of the peptide before we were ready meant a premature response and a new dish of cells. Nevertheless, we found that a short pulse of Wnt hyperpolarized the cells, and this could be traced to a small rise in a calcium-activated $\mathrm{K}$ channel (Fig. 6). The bioelectric signal was thus a very sensitive and fast indicator of Wnt activity, much more rapid than previous assays, but at a single cell level. Publishing such results can be interesting as cancer is often seen as a problem for molecular biologists or for clinicians only. A certain amount of mutual education is required to ensure that reviewers grasp the biophysics!

\section{What Can We Learn from Bioelectricity?}

I have tried here to give a sampler of a few topics? which can be accessed by measuring the bioelectrical signals from the cell membrane. The techniques are recognized as standard in neurobiology, although some of them take a while to acquire and, more importantly, the results need effort to interpret. However, there are still limiting technical problems when trying to measure ultrafast events. This is obvious in hearing where the biological signals extend to $100 \mathrm{kHz}$, yet many of the technologies (the patch clamp for example) have a recording bandwidth of $20 \mathrm{kHz}$ at most. More generally, an outstanding basic question is: how fast does an ion channel open? We can only guess at the answer using models, extrapolation, or "coarse grained" molecular dynamics.

There is little doubt that recording electrical signals from single cells requires a certain degree of dedication, but such hard won data can provide fundamental insights into biological processes on which industrial and medical advances may well depend. Understanding the dynamics and modulation of the electrical potentials across a functioning cell membrane is an essential complement to the progress of appreciating the structural, molecular, cellular, and systems properties of living systems. 


\section{References}

1. Baylor DA, Hodgkin A, Lamb TD. The electrical response of turtle cones to flashes and steps of light. J Physiol 1974;242:685-727. DOI: 10.1113/jphysiol.1974 .sp010731.

2. Russell IJ, Sellick PM. Intracellular studies of hair cells in the Mammalian Cochlea. J Physiol 1978;284:261-90. DOI: 10.1113/jphysiol.1978.sp012540.

3. Crawford AC, Fettiplace R. An electrical tuning mechanism in turtle cochlear hair cells. J Physiol 1981;312:377412. DOI: $10.1113 /$ jphysiol.1981.sp013634.

4. Corey DP, Hudspeth AJ. Ionic basis of the receptor potential in a vertebrate hair cell. Nature 1979;281:675677. DOI: $10.1038 / 281675 \mathrm{a} 0$.

5. Neher E, Sakmann B. Single-Channel Currents Recorded from Membrane of Denervated Frog Muscle Fibres. Nature 1976;260:799-802. DOI: 10.1038/260799a0.

6. Ashmore JF, Meech RW. Ionic basis of membrane potential in outer hair cells of guinea pig cochlea. Nature 1986;322: 368-371. DOI: 10.1038/322368a0.

7. Housley GD, Ashmore JF. Ionic currents of outer hair cells isolated from the guinea-pig cochlea. J Physiol 1992;448: 73-98. DOI: 10.1113/jphysiol.1996.sp021715.

8. Zheng J, Shen W, He DZ, et al. Prestin is the motor protein of cochlear outer hair cells. Nature 2000;405:149-155. DOI: $10.1038 / 35012009$.

9. Oliver D, He DZ, Klocker N, et al. Intracellular Anions as the Voltage Sensor of Prestin, the Outer Hair Cell Motor Protein. Science 2001;292:2340-2343. DOI: 10.1126/ science.1060939.
10. Mistrik P, Daudet N, Morandell K, et al. Mammalian prestin is a weak $\mathrm{Cl}(-) / \mathrm{HCO}(3)(-)$ electrogenic antiporter. J Physiol 2012;590:5597-5610. DOI: 10.1113/jphysiol.2012.241448.

11. Griesinger CB, Richards CD, Ashmore JF. Fast vesicle replenishment allows indefatigable signalling at the first auditory synapse. Nature 2005;435:212-215. DOI: 10.1038/ nature03567.

12. Ashmore JF, Falk G. The single-photon signal in rod bipolar cells of the dogfish retina. J Physiol 1980;300:151166. DOI: 10.1113/jphysiol.1980.sp013156.

13. Ashmore JF. Frequency tuning in a frog vestibular organ. Nature 1983;304:536-538. DOI: 10.1038/304536a0.

14. Mammano F, Ashmore JF. Differential expression of outer hair cell potassium currents in the isolated cochlea of the guinea-pig. J Physiol 1996;496:639-646. DOI: 10.1113/ jphysiol.1996.sp021715.

15. Gale JE, Ashmore JF. An intrinsic frequency limit to the cochlear amplifier. Nature 1997;389:63-66. DOI: 10.1038/37968.

16. Ashmore J, Olsen H, Sørensen N, et al. Wnts control membrane potential in mammalian cancer cells. J Physiol 2019;597:5899-5914. DOI: 10.1113/JP278661.

Address correspondence to:

Jonathan F. Ashmore, PhD

Department of Neuroscience, Physiology and Pharmacology

UCL Ear Institute, UCL London WC1E 6BT United Kingdom

Email: j.ashmore@ucl.ac.uk 